УРГЕНТНЫЕ ОПЕРАЦИИ ПРИ ЗАБОЛЕВАНИЯХ ЩИТОВИДНОЙ ЖЕЛЕЗЫ, ОСЛОЖНЕННЫХ КОМПРЕССИЕЙ ОРГАНОВ ШЕИ И СРЕДОСТЕНИЯ

Гостимский А.В. ${ }^{1}$, Романчишен А.Ф. ${ }^{1}$, Селиханов Б.А. ${ }^{1}$, Лисовский О.В. ${ }^{1}$

1 ФГБОУ ВО «Санкт- Петербургский государственный педиатрический медицинский университет» Минздрава России,194100, ул. Литовская 2, Санкт-Петербург, Россия;

Резюме. Сообщения о неотложных и срочных вмешательствах на щитовидной железе (ЩЖ) встречаются редко. Целью исследования явилась разработка и внедрение в практику оптимальную тактику лечения больных узловыми новообразованиями щитовидной железы, вызвавшими компрессию органов шеи и средостения.

С 1974 по 2015 годы в Центре хирургии органов эндокринной системы оперированы по неотложным или срочным показаниям 167 больных с патологией ЩЖ, что составило 0,57\% от 29325 пациентов. У всех больных были симптомы компрессионного синдрома.Все пациенты по срочности операции разделены на 2 группы. Первую группу составили 52 (31,1\%) пациента, перенесшие неотложные хирургические вмешательства. Во вторую группу включены 115 (68,9\%) больных, оперированных по срочным показаниям.

Средний возраст больных, оперированных по неотложным и срочным показаниям,

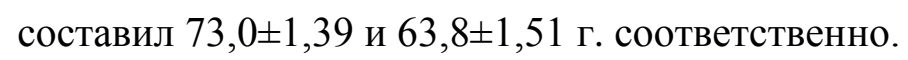

Среди причин, вызвавших компрессионный синдром, дифференцированный рак ЩЖ (ДРЩЖ) наблюдался в 79 (47,3\%) случаях. У 88 (52,7\%) больных экстренные хирургические вмешательства выполнены по поводу полинодозного эутиреоидного шейнозагрудинного зоба (ПЭШЗ3).

В работе нами впервые использовано определение степени сужения трахеи с помощью спирографии у 135 (80,84\%) больныхдля выявления сроков операции.

В ходе неотложных хирургических вмешательств радикальные операции выполнены 8 (22,9\%) больным ДРЩЖ, в случаях срочных вмешательств этот показатель в 2 раза больше -23 (52,3\%) пациента.

Выполнение срочных вмешательств увеличивает возможность радикальных хирургических вмешательств, уменьшая частоту послеоперационных осложнений.

Ключевые слова: щитовидная железа; неотложные и срочные операции; компрессия трахеи.

\title{
URGENT OPERATIONS IN PATIONS WITH DISEASES OF THYROID GLAND COMPLICATED BY COMPRESSION OF NECK AND MEDIASTINUM
}

Gostimskii A.V. ${ }^{1}$, Romanchishen A.F. ${ }^{1}$, Selikhanov B.A. ${ }^{1}$, Lisovskii O.V. ${ }^{1}$ 
${ }^{1}$ St. Peterburg State Pediatric Medical University of Ministry of Healthcare of Russian Federation, St. Petersburg, Russia

Summary. Reports of immediately and urgent interventions on the thyroid gland are rare. The aim of the study was to develop and put into practice the optimal tactics of treating patients with nodular tumors of the thyroid gland, which caused compression of the organs of the neck and mediastinum.

From 1974 to 2015, 97 patients with thyroid disease were operated in the Endocrine Surgery Center, they was $0.57 \%$ of 29325 patients. All patients had symptoms of compression syndrome. All patients were divided into 2 groups. I group consist of 52 (31.1\%) patient who underwent immediately surgical interventions. The second group included 115 (68.9\%) patients operated for urgent indications.

The mean age of patients operated for immediately reasons was $73.0 \pm 1.39$ year.The mean age of patients operated for urgent indications was $63.8 \pm 1.51$ year.

Among the reasons that caused the compression syndrome, the differentiated cancer of the thyroid gland was observed in $79(47.3 \%)$ cases. Benign thyroid diseases caused urgent surgical interventions in $88(52.7 \%)$ patients.

In our work, we first used the definition of the degree of tracheal constriction with spirography in $135(80.84 \%)$ patients to identify the timing of surgery.

In the course of emergency surgical interventions, radical operations were performed on 8 $(22.9 \%)$ patients with differentiatedcancer of the thyroid gland, in cases of urgent interventions, this figure is 2 times more - $23(52.3 \%)$ patients.

The performance of urgent operations increases the possibility of radical surgical interventions, reducing the frequency of postoperative complications.

Key words: thyroid gland; immediately and urgent operations; compression of the trachea.

Введение. Компрессионный синдром является одной из основных причин гибели больных запущенным раком щитовидной железы [1,2]. Реже показаниями к неотложным и срочным операциям являются кровотечение из опухолей $[3,4]$, сдавление пищевода с выраженной дисфагией [5].

Единых взглядов на показания к неотложным и срочным хирургическим вмешательствам у больных зобом нет. А.Г.Высоцкий и соавт. (2010) разработали показания к неотложным операциям у больных зобом шейно-загрудинной локализации, основанные на определении коэффициента обтурации верхней грудной апертуры, полученной по данным КТ органов шеи и средостения [6].А.В.Гостимский и соавт. (2013) рекомендуют использовать спирометрию для определения сроков операции при заболеваниях ЩЖ, осложненных компрессией трахеи [7]. 
Таким образом, проблема оказания хирургической помощи больным острыми расстройствами дыхания в результате сдавление трахеи в шейном и шейно-загрудинном отделе не решена и мало освещена.

Цель работы: разработать и внедрить в практику оптимальную тактику лечения больных узловыми новообразованиями щитовидной железы, вызвавшими компрессию органов шеи и средостения.

Материалы и методы. В работу включены анализ и результаты лечения 167 (0,57\%) пациентов, поступивших в Центр хирургии органов эндокринной системы г. СанктПетербурга в экстренном порядке с осложнениями заболеваний ЩЖ в период с 1974 по 2015 год. Все больные оперированы по неотложным и срочным показаниям.

Возраст оперированных больных был от 24 до 86 лет, составив в среднем $68,2 \pm 1,32$. Наиболее часто болели женщины - 155 (92,8\%) наблюдений, мужчин было всего 12 (7,2\%) больных. Соотношение женщин и мужчин выражалось как 13:1. Анамнез

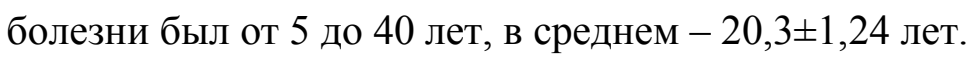

По срокам выполнения хирургических вмешательств все больные разделены на 2 группы. В I группу вошли 52 (31,1\%) пациент, которым выполнены хирургические вмешательства по неотложным показаниям в течение первых суток.ІІгруппу составили 115 (68,9\%) больных, оперированные по срочным показаниям в течение вторых и третьих суток с момента поступления в стационар.

В данной работе нами впервые использовано определение степени сужения трахеи с помощью спирографии. Определялся объем форсированного выдоха за одну секунду (ОФВ1) на спирографе МАС-1. Данный метод использовался в 135 (80,84\%) наблюдениях.

Результаты и обсуждение. Все пациенты, поступившие в стационар с компрессионным синдромом, угрозой асфиксии, наблюдались у эндокринолога и других

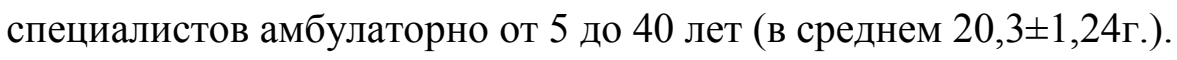

У больных Ігруппы в 25 (48,0\%) случаяхкомпрессию трахеи вызывал первичный ДРЩЖ, у 10 (19,3\%) - рецидивный ДРЩЖ и в 17 (32,7\%) случаях - ПЭШЗ3.

Причиной срочных операций во ІІруппе в 32 (27,8\%) наблюдениях был первичный ДРЩЖ, у 12 (10,5\%) больных рецидивный ДРЩЖ, у 55 (47,8\%) пациентов ПЭШЗ3, у 16 (13,9\%) больных рецидивный ПЭШЗ3.

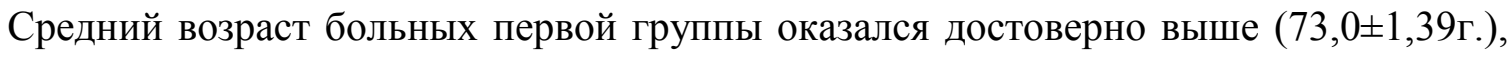
чем у пациентов второй группы $(63,8 \pm 1,51 г$. $)(\mathrm{p}<0,05)$.

Для определения сроков выполнения операции у пациентов с патологией ЩЖ, осложненной жизнеугрожающими состояниями в результате компрессии верхних дыхательных путей, нами разработан метод определения степени сужения трахеи с 
помощью спирографа (патент Российской Федерации №2533049 от 27.11.2013г). Данный метод обследования использован у 135 больных (80,84\%) за период с 2000 по 2015 годы.

При спирографическом исследовании оценивался показатель ОФВ1 как объективная оценка степень сужение трахеи. Если в спирограмме имеется значительное отклонение ОФВ 1 от нормы (35-54\%), больному показана срочная операция. При резких отклонениях от нормы (менее 35\%) показана неотложная операция.

По результатам спирографии в 34 (25,2\%) наблюдениях пациенты оперированы по неотложным показаниям, в 101 (74,8\%) случаях проведены срочные хирургические вмешательства. Следует отметить, что у 18 (13,3\%) пациентов, поступивших в стационар в тяжелом состоянии только определение значение ОФВ1 позволило отсрочить операцию. У данных больных длительная предоперационная подготовка позволила улучшить результаты хирургического вмешательства

У больных ДРЩЖ II группы радикальные операции выполнены в 8 наблюдениях, что составило 22,9\%. У пациентов Ігруппы этот показатель в 2 раза больше - у 23 (52,3\%) пациентов $(\mathrm{p}<0,05)$.

В раннем послеоперационном периоде осложнения выявлены у 11 (21,2\%) пациентов первой группы, что в 4 раза превысило аналогичный показатель больных второй группы $(\mathrm{p}<0,05)$.

Заключение. Таким образом, спирографическое обследование позволяет предотвратить задержку с хирургическим лечением у больных, нуждающихся в неотложной операции. Выявление пациентов, которым показаны срочные хирургические вмешательства, дало возможность более полной предоперационной подготовки и улучшения результатов хирургического лечения. Выполнение срочных вмешательств увеличивает возможность радикальных хирургических вмешательств у больных ДРЩЖ и уменьшает частоту послеоперационных осложнений.

\section{Список литературы:}

1. Романчишен А.Ф. Неотложные состояния в тиреоидной и паратиреоидной хирургии. СПб: ООО "Типография Феникс"; 2014.

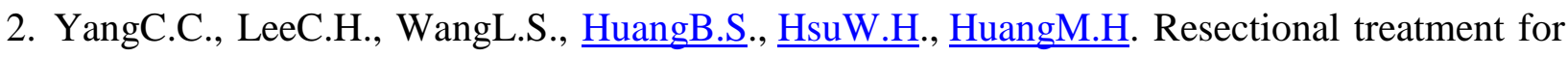
thyroid cancer with tracheal invasion: a long-term follow-up study. Arch Surg.2000; 135(6): 704707.doi:10.1001/archsurg.135.6.704

3. Kumar S., JoshiM.K. Emergency total thyroidectomy for bleeding anaplastic thyroid carcinoma: a viable option for palliation. Indian J. Palliat. Care. 2011; 17(1): 67-69.doi: $\underline{10.4103 / 0973-1075.78452}$ 
4. Testini M., Logoluso F., Lissidini G., Gurrado A., Campobasso G., Cortese R., De Luca G.M., Franco I.F., De Luca A., Piccinni G. Emergency total thyroidectomy due to non-traumatic disease. Experience of a surgical unit and literature review.World Journal of Emergency Surgery. 2012; 7:9. Available at:http://www.wjes.org/content/7/1/9(accessed: 12.10.2015). doi: 10.1186/1749-7922-7-9

5. MortonR.P., AhmadZ.Thyroid cancer invasion of neck structures: epidemiology, evaluation, staging and management. Current Opinion in Otolaryngology \& Head \& Neck Surgery. 2007; 15(7): 89-94.doi: 10.1097/MOO.0b013e3280147348

6. Высоцкий А.Г., Сидоренко Ю.А., Гюльмамедов С.И., Вегнер Д.В., КитовС.Ю. Критерии оценки синдрома шейно-медиастинальной компрессии при патологии щитовидной железы. Украинский журнал хирургии. 2010; 1: 44-46.

7. Гостимский А.В., Романчишен А.Ф., Селиханов Б.А.Неотложныеоперации на щитовидной железе. Онкохирургия. - 2013.- 5 (Спецвыпуск 1).- С. 152.

\section{Информация об авторах.}

Гостимский Александр Вадимович, Санкт-Петербургский Государственный педиатрический медицинский университет, д.м.н., профессор, тел. 992-29-70, Еmail:gostimsky@mail.ru

Романчишен Анатолий Филиппович, Санкт-Петербургский Государственный педиатрический медицинский университет, д.м.н., профессор, тел. 904-28-56,Е-mail: info@endocrinesurgery.ru

Селиханов Бахрам Атамуратович, Санкт-Петербургский Государственный педиатрический медицинский университет, ассистент, тел. 8-961-802-24-09,E-mail: bahram83@ mail.ru Лисовский Олег Валентинович, Санкт-Петербургский Государственный педиатрический медицинский университет,к.м.н., доцент, тел. 8-921-390-88-91,E-mail: oleg.lisovsky@rambler.ru 